\title{
Molecular rotors with designed polar rotating groups possess mechanics-controllable wide-range rotational speed
}

\author{
Jian Shao $\mathbb{D}^{1,2}$, Wenpeng Zhu ${ }^{1,2}$, Xiaoyue Zhang $\mathbb{D}^{1,2 凶}$ and Yue Zheng $\mathbb{D}^{1,2 \bowtie}$
}

\begin{abstract}
Molecular rotors with controllable functions are promising for molecular machines and electronic devices. Especially, fast rotation in molecular rotor enables switchable molecular conformations and charge transport states for electronic applications. However, the key to molecular rotor-based electronic devices comes down to a trade-off between fast rotational speed and thermal stability. Fast rotation in molecular rotor requires a small energy barrier height, which disables its controllability under thermal excitation at room temperature. To overcome this trade-off dilemma, we design molecular rotors with co-axial polar rotating groups to achieve widerange mechanically controllable rotational speed. The interplay between polar rotating groups and directional mechanical load enables a "stop-go" system with a wide-range rotational energy barrier. We show through density functional calculations that directional mechanical load can modulate the rotational speed of designed molecular rotors. At a temperature of $300 \mathrm{~K}$, these molecular rotors operate at low rotational speed in native state and accelerates tremendously (up to $10^{19}$ ) under mechanical load.
\end{abstract}

npj Computational Materials (2020)6:185; https://doi.org/10.1038/s41524-020-00457-6

\section{INTRODUCTION}

Artificial molecular rotors are attracting increasing interests for their potential applications in molecular machines and nanomechanical electronic devices ${ }^{1}$. Mimicking biological molecular rotors and macroscopic mechanical rotors, artificial counterparts transducing light ${ }^{2-6}$, heat ${ }^{7,8}$, or electrical stimuli ${ }^{9,10}$ into mechanical rotation are designed and produced. Structural design for molecular rotors, including stator (static group) and rotor (rotating group) ${ }^{11}$, enables desirable functionality ${ }^{12}$ in molecular scale, such as motor ${ }^{13}$, propeller ${ }^{14-16}$, switch ${ }^{17,18}$ and sensor ${ }^{19}$. Especially, molecular rotors convert conformation during rotational reactions, which switches electronic properties and thus emerges as promising electronic devices ${ }^{20}$.

For application in molecular devices, molecular rotors require both fast rotational speed and thermal stability. According to transition state theory, however, there is a trade-off between rotational speed $k$ and thermal stability (represented by free energy barrier):

$k \propto \frac{k_{\mathrm{B}} T}{h} \mathrm{e}^{-\Delta \mathrm{G}^{\ddagger} / k_{\mathrm{B}} T}$,

where $k_{\mathrm{B}}$ is Boltzmann's constant, $T$ is the temperature, $h$ is Planck's constant, and $\Delta G^{\ddagger}$ represents the standard free energy barrier (or Gibbs free energy of activation). For a molecular rotor, fast rotational speed and thermal stability cannot exist at the same time. This comes down to a trade-off dilemma in designing molecular rotors: accelerating rotational speed leads to reducing of thermal stability, and vice versa $2,3,21,22$.

To solve the trade-off dilemma, two possible means are employed $^{23}$. One means is to induce transient excitation on molecular rotor by electrical field ${ }^{24}$ or light stimulus ${ }^{4}$, which is widely employed in over-crowded molecular motor systems to overcome large energy barriers. An alternative way is to control rotating speed continuously by applying "brake-throttle" systems ${ }^{25}$ on the rotational energy barrier. For example, 100 times change of rotational speed is achieved through electrical bias ${ }^{17}$; control of rotational frequency between range of $10^{3}-10^{8} \mathrm{~Hz}$ is reported ${ }^{26}$ in continuously changing lattice through solid-solution approach; $\mathrm{Li}+$ concentration modulates rotational speed from $1.3 \times 10^{-2} \mathrm{~Hz}$ to $2.3 \mathrm{~Hz}$ continuously ${ }^{27}$. For application in modern electronic devices, molecular rotors require even larger tuning range: operations require rotational speed at Gigahertz $\left(10^{9} \mathrm{~Hz}\right)$ while thermal stability requires rotation ceased as long as possible, e.g., one day $\left(10^{-6} \mathrm{~Hz}\right)$. To cover a rotational frequency range $\left(k_{1}\right.$, $k_{2}$ ) at a given temperature, free energy barrier should be tuned as

$\Delta G_{1}^{\ddagger}-\Delta G_{2}^{\ddagger} \propto k_{\mathrm{B}} T \ln \frac{k_{2}}{k_{1}}$.

In other words, to achieve a specific range of controllable rotational speed, corresponding tuning range of energy barrier increases linearly with temperature. For example, the required range of controllable energy barrier at $300 \mathrm{~K}$ is 75 times the one at $4 \mathrm{~K}$. Consequently, working temperature of molecular rotors is largely limited by its controllable range of energy barrier. Considering the demand of utilizing molecular rotors at room temperature ${ }^{28-30}$, there is a need to explore means to control energy barrier continuously and effectively.

To achieve wide-range rotational speed at room temperature, we design a series of anthracene-based molecular rotors with rotational energy barrier ultra-sensitive to directional pressure. We use directional mechanical force as the accelerator to push coaxial polar rotors into flip, which modulates energy barrier efficiently. With directional nature of polar rotor, the rotors "stop" with high barriers and "go" in low barriers under variable directional mechanical loads. As a result, the acceleration of rotational speed of an investigated molecular rotor exceeds up to 19 orders of magnitude at $300 \mathrm{~K}$. Through substituting different polar rotors, modulation of different ranges of rotational speed can be achieved.

${ }^{1}$ State Key Laboratory of Optoelectronic Materials and Technologies, Sun Yat-sen University, 510275 Guangzhou, China. ${ }^{2}$ Physical Mechanics and Biophysics Research Center, School of Physics, Sun Yat-sen University, 510275 Guangzhou, China. ${ }^{凶}$ email: zhangxy26@mail.sysu.edu.cn; zhengy35@mail.sysu.edu.cn 


\section{RESULTS}

Design and energy barrier of molecular rotors

We design in Fig. 1a six molecular rotors (MR) 1-6 with anthracene as the stator. To achieve a different range of controllable rotational speed, different polar groups, i.e., pyridine, thiophene, 3,4-difluorothiophene, 3-thiophenecarboxaldehyde, thiophenol and 3-cyanothiophene, are attached to position 9 and 10 of anthracene as co-axial polar rotors (shown in Fig. 1a with red skeletal formula). For MR1, the torsion angle between anthracene and pyridine is ca. $71^{\circ}$. For other molecular rotors, anthracene and polar rotors are perpendicular. Polarity of each polar rotor attached to anthracene stator produces two distinct equilibrium states denoted in Dirac notation: $|\uparrow\rangle$ and $|\downarrow\rangle$. Here each arrow denotes the dipole moment of the polar group and only plus-minus of dipole moment perpendicular to anthracene plane is accounted. As two polar rotors are attached to anthracene, the state of a molecular rotor can be denoted as $|\uparrow \uparrow\rangle,|\downarrow \downarrow\rangle$ and $|\uparrow \downarrow\rangle$. In gas phase, $|\uparrow \uparrow\rangle$ and $|\downarrow \downarrow\rangle$ are degenerate. Due to polarity of the rotor, the energies of $|\uparrow \uparrow\rangle$ and $|\uparrow \downarrow\rangle$ are slightly different (see Supplementary Table 1). For simplicity, $|\uparrow \uparrow\rangle$ is used as reactant in the following investigations. Transition state (TS) is denoted as $|\rightarrow\rangle$ although the dipole moment of the rotor perpendicular to anthracene plane is not strictly zero.

We show in Fig. 1b the free energy barrier $\Delta G^{\ddagger}$ for rotational reaction $|\uparrow \uparrow\rangle$ to $|\uparrow \downarrow\rangle$ through $|\uparrow \rightarrow\rangle$ of MR1-6 together with their corresponding reaction rate at $300 \mathrm{~K}$. For rotation of a polar rotor, two TS are possible: $|\uparrow \rightarrow\rangle$ and $|\downarrow \rightarrow\rangle$ (see Supplementary Table 2). However, the difference of $\Delta G^{\ddagger}$ through these two TS is relatively small (see Supplementary Table 3). Therefore, we pick TS shown in Fig. 1a for further analysis and define them as $|\uparrow \rightarrow\rangle . \Delta G^{\ddagger}$ ranges from ca. $72 \mathrm{~kJ} \mathrm{~mol}^{-1}$ (MR2) to ca. $171 \mathrm{~kJ} \mathrm{~mol}^{-1}$ (MR5), indicating significant influence by the structure of polar rotor. To elaborate, we follow Taft equation, which separates the effects of structure on free energy barrier to the sum of independent steric and polar effects $^{31}$. For steric effects, we employ noncovalent interaction ${ }^{32}$ $(\mathrm{NCl})$ analysis (shown in Supplementary Fig. 1) on TS of the molecular rotors. During the rotation, polar rotors endure different steric hindrances around the axis connecting position 9 and 10 of anthracene stator. Among the molecular rotors, MR5 has the largest area of steric hindrance between polar rotor and stator, which produces the highest free energy barrier. Moreover, the steric effects are considered beyond simple length-dependent rules. While MR6 has longer polar rotors than MR4 (ca. $127 \mathrm{~kJ}$ $\mathrm{mol}^{-1}$ ) and MR5 (ca. $171 \mathrm{~kJ} \mathrm{~mol}^{-1}$ ), it has a much lower free energy barrier of ca. $105 \mathrm{~kJ} \mathrm{~mol}^{-1}$. It is because steric hindrance from the anthracene stator is mainly from the hydrogen atom near position 9 or 10. In gas phase, the distance between two carbon atoms in position 9 and 1 is $2.48 \AA$. Therefore, the length of polar rotor excessing this threshold distance has little contribution to steric hindrance.

The steric hindrance between different polar rotors and the stator brings complicated effects on path of rotational reaction. Intrinsic reaction coordinate (IRC) calculation in Supplementary Figs. 2-7 gives a comprehensive view on reaction path $|\uparrow\rangle$ to $|\downarrow\rangle$ for one of the co-axial rotors (while the other rotor is in $|\uparrow\rangle$ ). From a conformational view, the movements of all six rotors have similar behaviors during the rotation process. Starting from $|\uparrow\rangle$, the polar rotor rotates around the axis connecting position 9 and 10 in anthracene stator with the change of dihedral angle between the stator and the rotor. When the dihedral angle decreases gradually and the polar rotor is near to the hydrogen atom on anthracene, the steric hindrance prohibits the rotation to some extent. To continue rotation, the connection between the rotor and the stator will bend to reach TS $|\rightarrow\rangle$.

For polar rotors, polar effects are also important. To elucidate this mechanism, $|\uparrow \rightarrow\rangle$ state with its electrostatic potential map is shown in Fig. 1a next to the skeletal formula. Polar rotor with larger dipole moment induces larger electrostatic potential value on the map and electrostatic interactions are inversely distancerelated. In the equilibrium state, the polar groups are far from the hydrogen atoms on positions 1 and 4 (or positions 5 and 8) of anthracene, so electrostatic interactions are weak. During the reaction, rotation of polar group decreases this distance and electrostatic interactions become strong. In TS, electrostatic interactions induce torsions on rotors related to the dipole moment vector of the polar rotor. For MR1, the net electrostatic interactions are positive, which increases the energy barriers; for

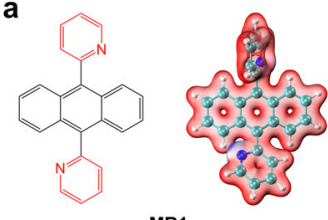

MR1

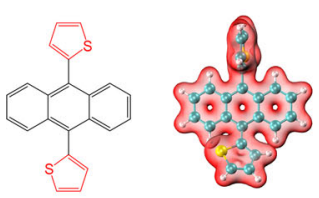

MR2

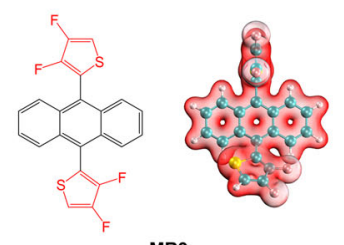

MR3

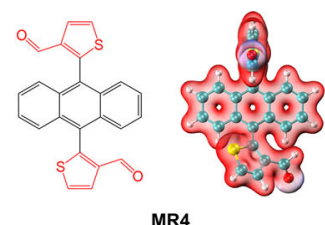

MR4

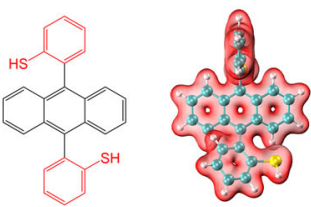

MR5

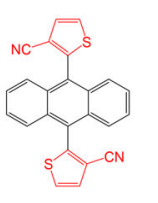

MR6 b

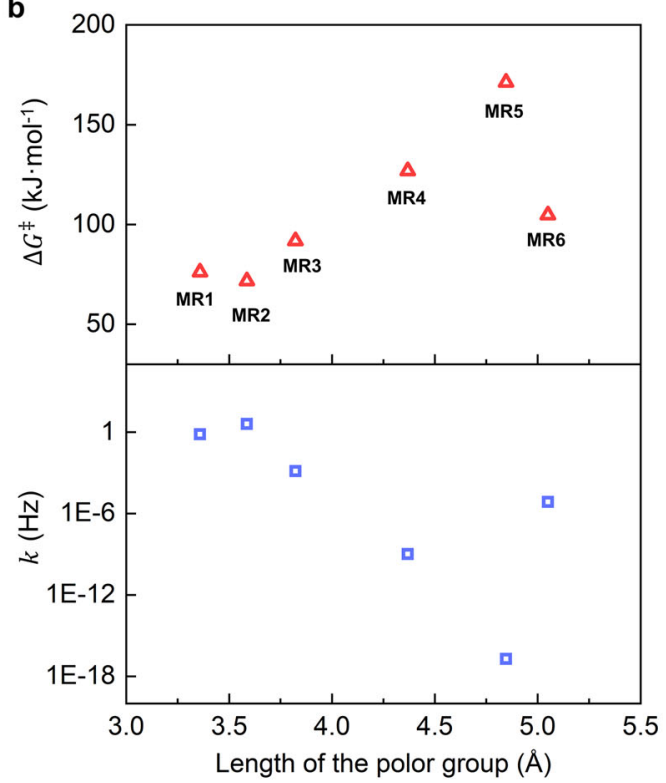

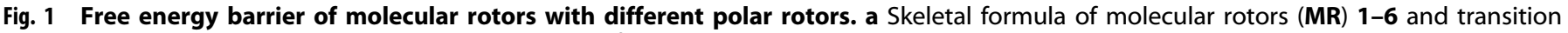

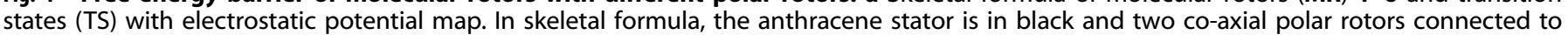

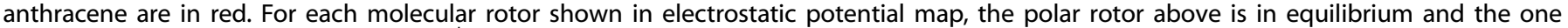
below is in TS. b Free energy barrier $\Delta G^{\ddagger}$ and rotational frequency $k$ for different molecular rotors. 
a
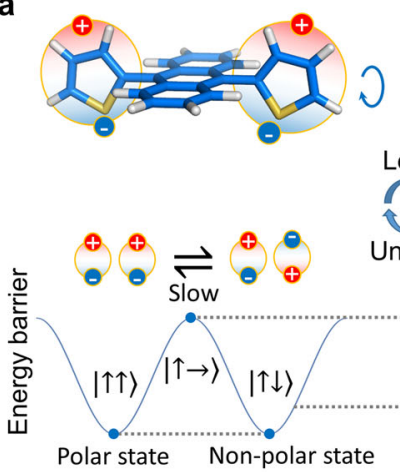

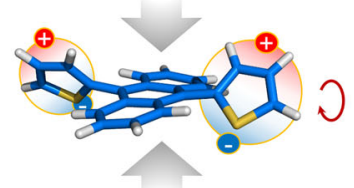

$\cap$

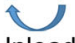

Unload

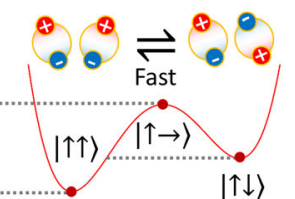

$|\uparrow \downarrow\rangle$

Polar state Non-polar state

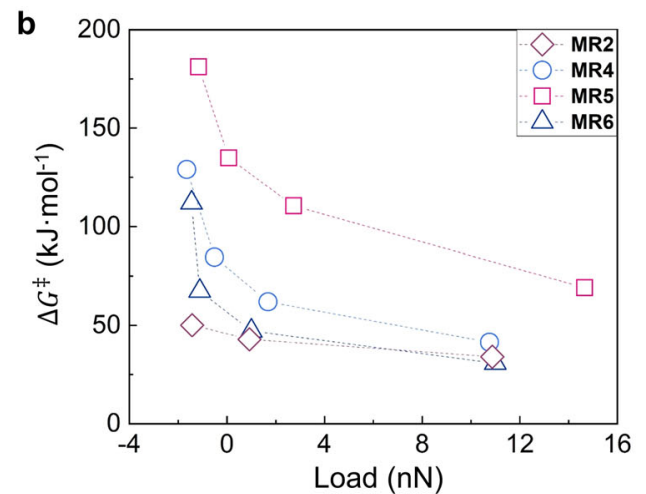

Fig. 2 Load-modulated free energy barrier for different molecular rotors. a Illustration of directional mechanical load on MR2 and its reversible controllability on free energy barrier of thiophenyl group. The dipole moment is denoted with pair of positive and negative signs. Applying load enables smaller free energy barrier and faster flip of thiophenyl group. Combination of both thiophenyl groups creates a "stopgo" system with polar and non-polar bi-states in MR2. b Free energy barrier for MR2, MR4, MR5, and MR6 from polar state to non-polar state under directional mechanical load.

MR6, the net electrostatic interactions are negative, which reduces the energy barrier significantly. For other molecular rotors, the dipole moment is relatively small (as shown in electrostatic potential map), so polar effects are not significant. Analysis in the second factor confirms the effects of dipole moment of rotors on rotational energy barriers.

As shown in Fig. 1b and Supplementary Fig. 8, the electronic energy barrier $\Delta E^{\ddagger}$ between $|\uparrow \uparrow\rangle$ and $|\uparrow \rightarrow\rangle$ from DFT calculation and the free energy barrier $\Delta G^{\ddagger}$ have similar trends with respect to the length of polar groups. This is expected because vibrational analysis for these molecular rotors gives similar ratios of partition functions in transition state and equilibrium state, i.e., $Q^{T S} / Q^{R}$. These polar groups cover rotational speed ranging from $10^{-17}$ to $10^{1} \mathrm{~Hz}$, which is too slow for electronic switch. In this regard, we will show in next section their acceleration in rotational speed through mechanical loading.

Control over rotational speed of polar rotors

To accelerate rotational speed, it is straightforward to reduce the rotational free energy barrier $\Delta G^{\ddagger}$. Mechanical force perpendicular to anthracene stator can largely modulated $\Delta G^{\ddagger}$ by changing the conformations of the molecular rotors. In Fig. 2a, we show illustration of reversible mechanical control of conformation and $\Delta G^{\ddagger}$ of MR2. Under the directional force applied through intermolecular interaction (see Supplementary Fig. 9), the dihedral angles between anthracene and polar groups are "squeezed" smaller. Therefore, $|\uparrow \uparrow\rangle$ and $|\uparrow \downarrow\rangle$ states are pushed nearer to TS $|\uparrow \rightarrow\rangle$, which reduces $\Delta G^{\ddagger}$.

The change of free energy barrier $\Delta G^{\ddagger}$ under mechanical load is shown in Fig. $2 b$ and indicates two features. First, when the mechanical force increases, $\Delta G^{\ddagger}$ for MR2, MR4, MR5, and MR6 decrease following a hyperbolic shape. Second, the reduction of $\Delta G^{\ddagger}$ varies for different polar groups. For MR2, $\Delta G^{\ddagger}$ decreases slowly from ca. $50 \mathrm{~kJ} \mathrm{~mol}^{-1}$ under $-1.43 \mathrm{nN}$ to ca. $34 \mathrm{~kJ} \mathrm{~mol}^{-1}$ under $10.87 \mathrm{nN}$. The change in $\Delta G^{\ddagger}$ is ca. $16 \mathrm{~kJ} \mathrm{~mol}^{-1}$. The effects of mechanical force on MR4-6 are more significant and rapid compared with MR2 (see Table 1). For MR4, $\Delta G^{\ddagger}$ decreases from ca. $129 \mathrm{~kJ} \mathrm{~mol}^{-1}$ under $-1.36 \mathrm{nN}$ to ca. $41 \mathrm{~kJ} \mathrm{~mol}^{-1}$ under $10.76 \mathrm{nN}$. The change in $\Delta G^{\ddagger}$ is ca. $88 \mathrm{~kJ} \mathrm{~mol}^{-1}$. For MR5, $\Delta G^{\ddagger}$ decreases from ca. $179 \mathrm{~kJ} \mathrm{~mol}^{-1}$ under $-1.17 \mathrm{nN}$ to ca. $75 \mathrm{~kJ} \mathrm{~mol}^{-1}$ under $14.66 \mathrm{nN}$. The change in $\Delta G^{\ddagger}$ is ca. $104 \mathrm{~kJ} \mathrm{~mol}^{-1}$. For MR6, free energy barrier decreases from ca. $107 \mathrm{~kJ} \mathrm{~mol}^{-1}$ under $-1.45 \mathrm{nN}$ to ca. $30 \mathrm{~kJ} \mathrm{~mol}^{-1}$ under $11.00 \mathrm{nN}$. The change in $\Delta G^{\ddagger}$ is ca. $77 \mathrm{~kJ} \mathrm{~mol}^{-1}$. In the region with negative force, mechanical effects on $\Delta G^{\ddagger}$ are more significant than in region with positive force.
Table 1. Maximum and minimum free energy barrier $\Delta G^{\ddagger}$ and rotational speeds $k$ at $300 \mathrm{~K}$ of molecular rotors under mechanical load from points in Fig. $2 b$.

\begin{tabular}{lllll}
\hline Molecular rotor & $\begin{array}{l}\text { Maximum } \\
\begin{array}{l}\Delta G^{\ddagger}(\mathrm{kJ} \\
\left.\mathrm{mol}^{-1}\right)\end{array}\end{array}$ & $\begin{array}{l}\text { Minimum } \\
k(\mathrm{~Hz})\end{array}$ & $\begin{array}{l}\text { Minimum } \\
\Delta G^{\ddagger}(\mathrm{kJ} \\
\left.\mathrm{mol}^{-1}\right)\end{array}$ & $\begin{array}{l}\text { Maximum } \\
k(\mathrm{~Hz})\end{array}$ \\
\hline MR2 & 50.1 & $2.4 \times 10^{4}$ & 34.0 & $1.5 \times 10^{7}$ \\
MR4 & 129.0 & $4.3 \times 10^{-10}$ & 41.4 & $7.9 \times 10^{5}$ \\
MR5 & 181.1 & $3.7 \times 10^{-19}$ & 69.1 & $1.2 \times 10^{1}$ \\
MR6 & 112.0 & $4.0 \times 10^{-7}$ & 30.7 & $5.6 \times 10^{7}$ \\
\hline
\end{tabular}

Rotational speed at a temperature of $300 \mathrm{~K}$ is calculated from Eq. (4) and shown in Table 1. Significant acceleration is achieved under mechanical load. Among these molecular rotors, MR5 has the largest acceleration from ca. $3.7 \times 10^{-19} \mathrm{~Hz}$ to ca. $1.2 \times 10^{1} \mathrm{~Hz}$ with a ratio of more than $10^{19}$. MR2 has the smallest acceleration from ca. $2.4 \times 10^{4} \mathrm{~Hz}$ to ca. $1.5 \times 10^{7} \mathrm{~Hz}$ with a ratio of $10^{2}$. MR6 has an acceleration from ca. $4.0 \times 10^{-7} \mathrm{~Hz}$ to ca. $5.6 \times 10^{7} \mathrm{~Hz}$. That is suitable $\Delta G^{\ddagger}$ for switch at room temperature: stable when load is weak and rotating fast under a load of $11 \mathrm{nN}$. Thus, acceleration in rotational speed in anthracene-based molecular rotors is achieved through directional mechanical load.

\section{Electronic energy difference among polarity states}

As shown in Fig. 2a, mechanical force not only reduces $\Delta G^{\ddagger}$, but also induces asymmetry of conformational distribution due to the polarity of the two co-axial rotors. Distinguishing the asymmetry is helpful in understanding the role of polar rotors in $\Delta G^{\ddagger}$ of molecular rotors. Polarity of two co-axial rotors attached to anthracene stator produces three distinct equilibrium states: $|\uparrow \uparrow\rangle$, $|\downarrow \downarrow\rangle$ and $|\uparrow \downarrow\rangle$. At gas phase (free standing), $|\uparrow \uparrow\rangle$ and $|\downarrow \downarrow\rangle$ are degenerate and have slight electronic energy difference with $|\uparrow \downarrow\rangle$ (see Supplementary Table 1). When directional load is applied, the angle between stator and rotor deviates from $90^{\circ}$. Clockwise or anticlockwise rotation of each polar rotor leads to molecular rotor with different configurations of polar groups. Each polarity state $(|\uparrow \uparrow\rangle,|\downarrow \downarrow\rangle$ and $|\uparrow \downarrow\rangle)$ splits into configurations with polar groups rotate in the same and opposite directions (observe through the line connecting positions 9 and 10). For a homogeneous load, these configurations can be denoted with point groups: $C_{2}$ or $C_{s}$ for $|\uparrow \uparrow\rangle$ and $C_{i}$ or $C_{2}$ for $|\uparrow \downarrow\rangle$. Among all the states, polar groups rotate in opposite direction of $|\uparrow \uparrow\rangle$ with $C_{2}$ symmetry has the 
lowest electronic energy, so we pick it as initial guess for further investigation.

Then we investigate the interaction between polar rotors and surfaces. The electronic energy difference for different polarity states is observed when spatial symmetry is broken by absorbing molecular rotors onto surface. We take MR2 as an example and absorb it onto $\mathrm{Au}(111)$ (see Fig. 3a). Three possible configurations $(|\uparrow \uparrow\rangle,|\downarrow \downarrow\rangle$ and $|\uparrow \downarrow\rangle)$ are accounted and their adsorption energies are calculated from DFT. Adsorbing MR2 onto surface will break the symmetry and energies of degenerate states split with $E_{|\downarrow \downarrow\rangle}>E_{|\uparrow \downarrow\rangle}>E_{|\uparrow \uparrow\rangle} \cdot|\uparrow \uparrow\rangle$ is the most stable configuration for MR2 on $\mathrm{Au}(111)$. For interaction between thiophene and $\mathrm{Au}(111)$, sulfur side has larger absorption energy than hydrogen side. Different studies $^{33-39}$ confirm that Au-S configuration in this situation is
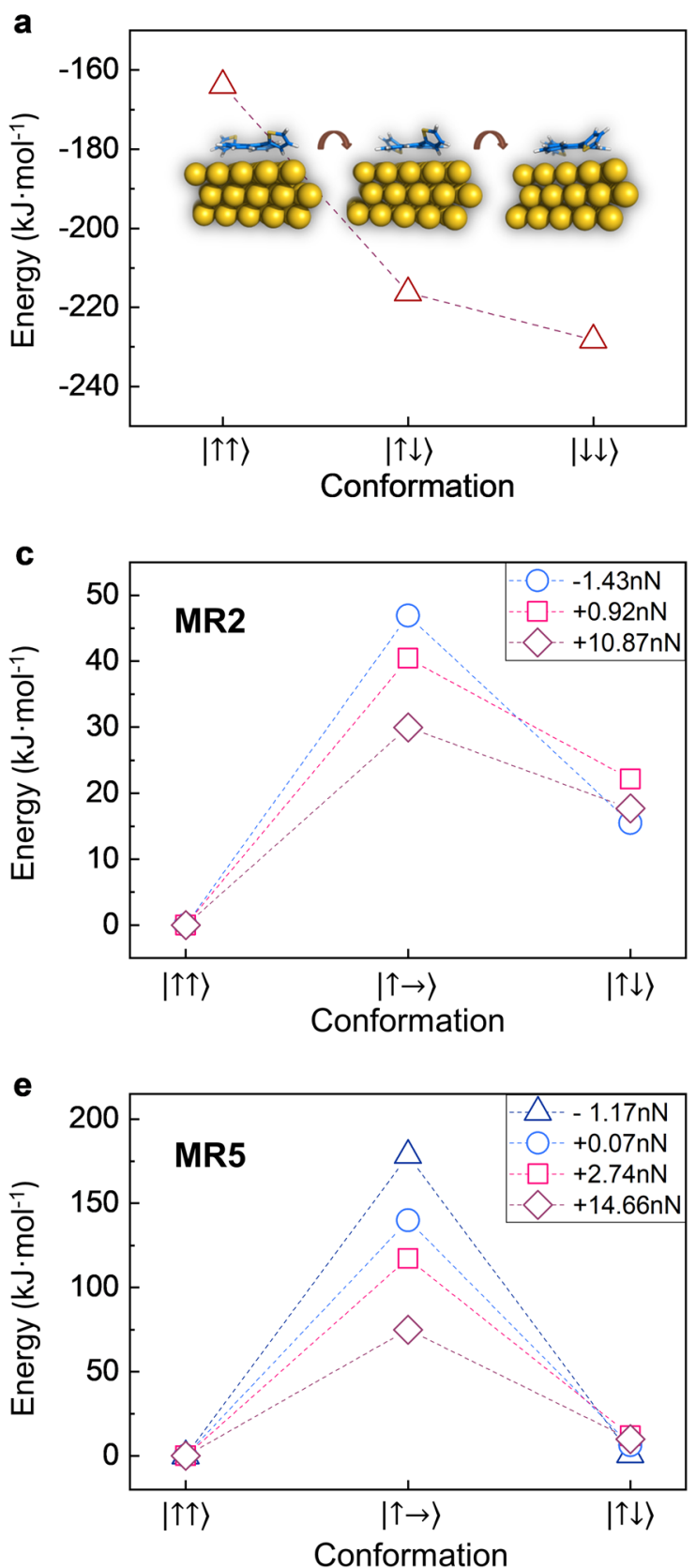

much more stable than Au-H. However, the electronic energy differences through flips of the two rotors are not equal. That is, flip from $|\downarrow \downarrow\rangle$ to $|\uparrow \downarrow\rangle$ has larger electronic energy difference than flip from $|\uparrow \downarrow\rangle$ to $|\uparrow \uparrow\rangle$.

Finally, the interaction between polar rotors and mechanical load is investigated. Electronic energy difference among different polarity states can also be observed when molecular rotor is under mechanical load. That is, $|\uparrow \uparrow\rangle$ and $|\downarrow \downarrow\rangle$ are degenerate, but $|\uparrow \uparrow\rangle$ and $|\uparrow \downarrow\rangle$ are not. Differences of $E_{|\uparrow \downarrow\rangle}$ and $E_{|\uparrow \uparrow\rangle}$ of MR2, MR4, MR5, and MR6 are force-dependent and the largest difference appears under load in the range of $0-3 \mathrm{nN}$ (see Fig. 3b). This can be attributed to deformation of anthracene stator under mechanical load. To note, this deformation preserves the point-group symmetry of the molecular rotors. Taking MR2 as an example
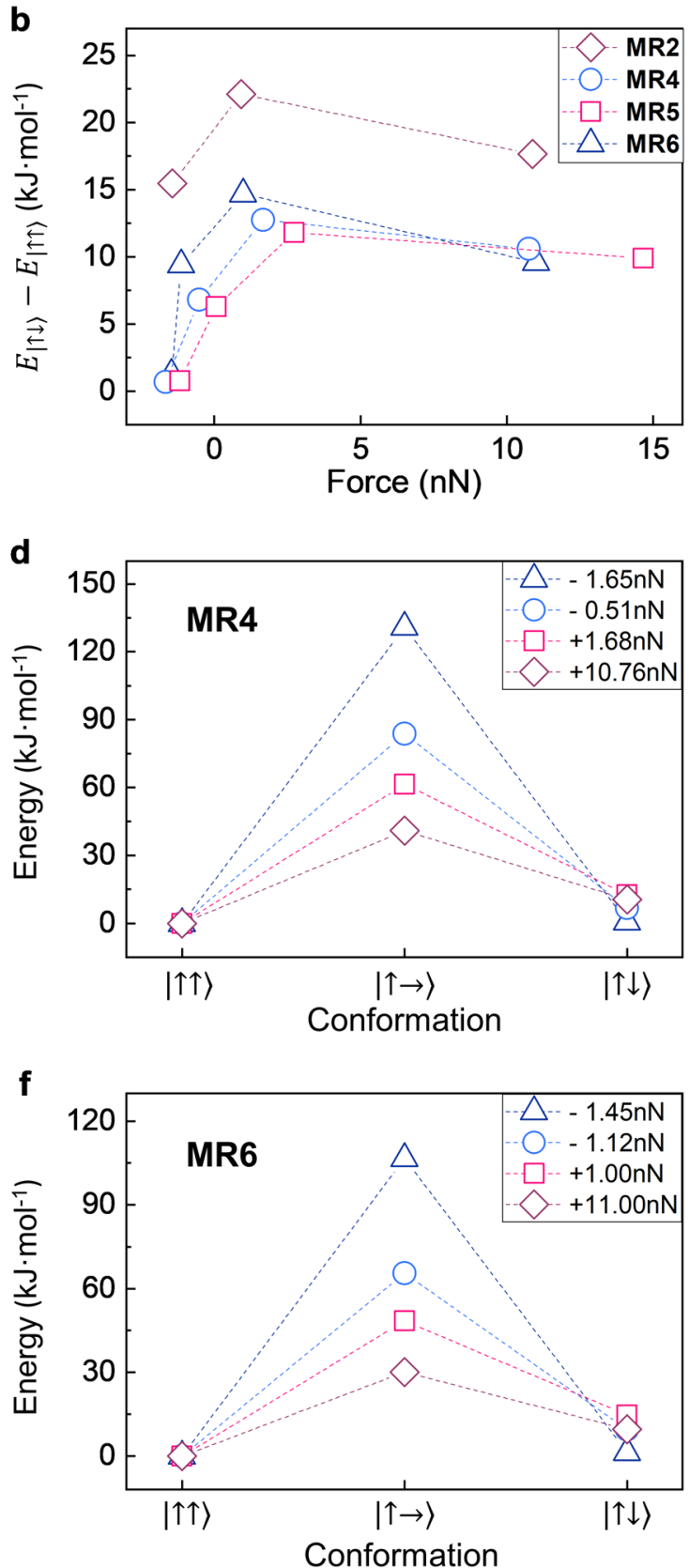

Fig. 3 Electronic energy difference of molecular rotor in polar and non-polar state. a Adsorption energy of three possible conformations of MR2 on $\mathrm{Au}(111)$. b Electronic energy difference between $|\uparrow \uparrow\rangle$ and $|\uparrow \downarrow\rangle$, i.e., $E_{|\uparrow \downarrow\rangle}-E_{|\uparrow \uparrow\rangle}$ of MR2, MR4, MR5 and MR6 under directional

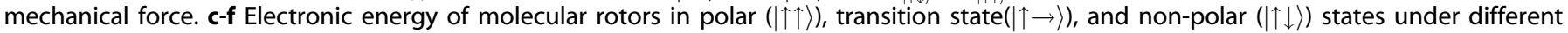
mechanical forces. 
(see Fig. 4), anthracene group in $|\uparrow \uparrow\rangle$ is in saddle-shaped distortion while it is tilted and without such distortion in $|\uparrow \downarrow\rangle$. The deformation of anthracene is quantified by deviation from the central plane. For $|\uparrow \uparrow\rangle$, displacements of upper and lower pairs of carbon atoms (on positions 7 and 6, 8 and 5, etc.) are different and even opposite under mechanical load $-1.43 \mathrm{nN},+0.92 \mathrm{nN}$, and $+10.87 \mathrm{nN}$. For $|\uparrow \downarrow\rangle$, displacements for both rows are in the same direction. These two modes of deformations lead to nondegeneration. Electronic nergy difference between $|\uparrow \uparrow\rangle$ and $|\uparrow \downarrow\rangle$ in Fig. $3 \mathrm{~b}$ is in accordance with the amplitude of deformation in anthracene, which increases from $-0.71 \mathrm{nN}$ to $+0.92 \mathrm{nN}$ and decreases from $+0.92 \mathrm{nN}$ to $+10.87 \mathrm{nN}$ (see Fig. $4 \mathrm{c}$ ). As a result, mechanical load pushes MR2 into $|\uparrow \uparrow\rangle$ instead of $|\uparrow \downarrow\rangle$. Similar results can be observed in MR4-6 (see Supplementary Figs. 15-17). The energies of these rotors in polar, non-polar and transition state are shown explicitly in Fig. 3c-f. Molecular rotor with larger energy barrier tends to have smaller electronic energy difference between $|\uparrow \uparrow\rangle$ and $|\uparrow \downarrow\rangle$ under the same load. This can also be attributed to distortion of anthracene stator under directional mechanical load. Larger energy barrier will hinder the rotation of rotors at the same load. Therefore, distortion reduces in $|\uparrow \uparrow\rangle$ and electronic energy difference between $|\uparrow \uparrow\rangle$ and $|\uparrow \downarrow\rangle$ is smaller. This phenomenon can be useful for rotational synchronization or assembling disordered multi-state molecules into ordered array through the mechanical load. We show a simple example of synchronized rotation of polar rotors in the following section.

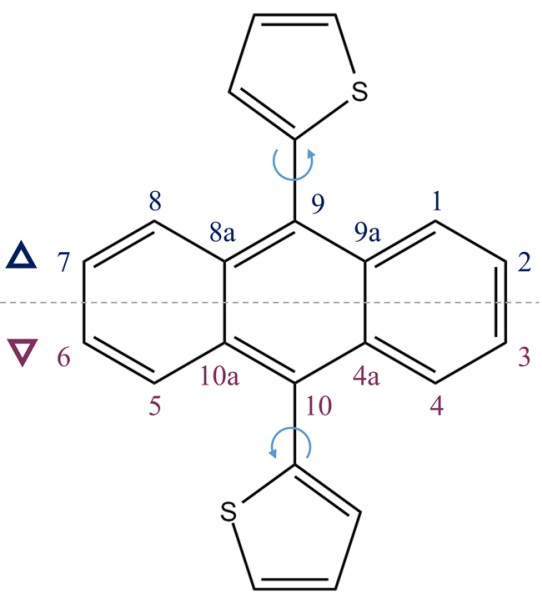

9,10-di(thiophen-2-yl)anthracene b

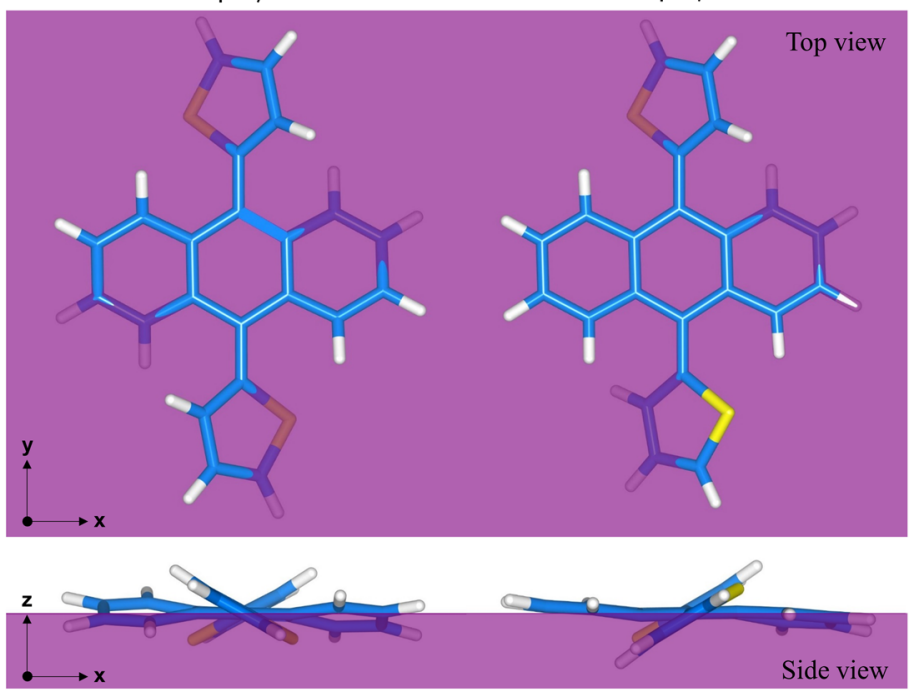

c

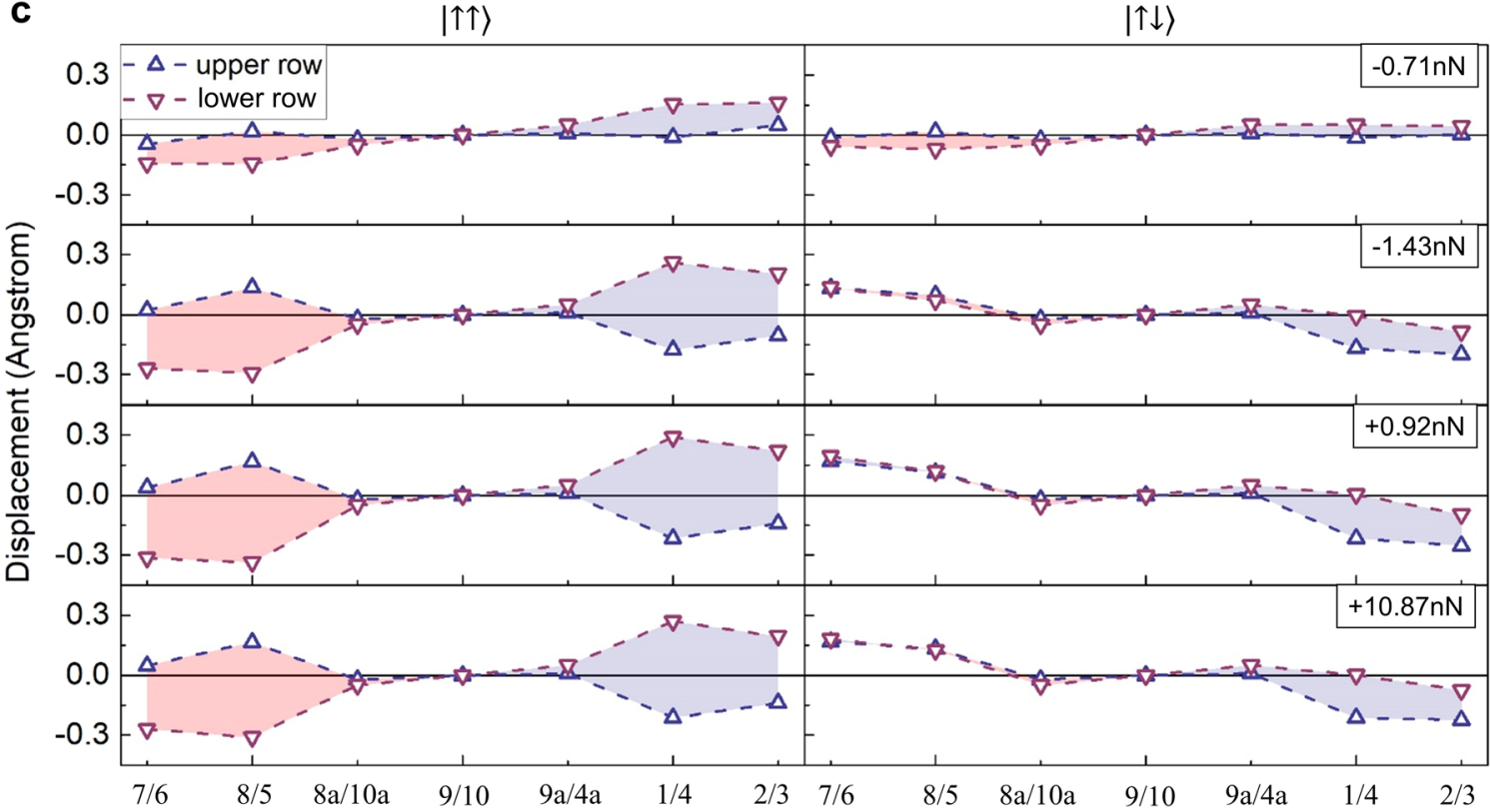

Fig. 4 Structural insights for electronic energy difference in polar and non-polar state. a Structure and atomic positions of MR2. b Conformation of MR2 in $|\uparrow \uparrow\rangle$ and $|\uparrow \downarrow\rangle$ states under $0.92 \mathrm{nN}$ of mechanical force. To illustrate the distortion of MR2, stick representation of the molecule is placed in purple "ink". The level of the "ink" crosses the central plane the molecular and structures lower than this level is immersed. c Displacement of carbon atoms in $\mathbf{z}$ direction from central plane corresponding to positions in a under directional mechanical loads for $|\uparrow \uparrow\rangle$ and $|\uparrow \downarrow\rangle$ states. The color in background indicates the difference in displacements of corresponding atom pairs: red for positive and blue for negative. 


\section{Rotational dynamics}

The acceleration and conformational selection of MR2 are verified by dipole moment rotation process through density functional tight-binding molecular dynamics (DFTB-MD) simulation (see Supplementary Movie 1). We apply mechanical forces by pushing two electrodes with MR2 in between (see Fig. 5a). To accelerate the process, we choose the temperature of $500 \mathrm{~K}$. Two loading schemes are applied and shown in Fig. 5b: the electrodes approach $0.2 \AA$ stepwise every 2 ps from $7.3 \AA$ to $6.7 \AA$ and to $6.5 \AA$, respectively, and then retreat. In each stage, the system is stabilized with RMSD evaluation (see Supplementary Fig. 18). In the first scheme, electrodes retreat from $6.7 \AA$ and dipole moment of the rotor stays positive throughout the loading process (see Fig. 5c). In the second scheme, electrodes approach further to $6.5 \AA$ to increase load on MR2 and the dipole moment of MR2 initiates to flip (see Fig. 5d). During the flip, orientations of both polar rotors are inclined to be parallel $(|\uparrow \uparrow\rangle$ or $|\downarrow \downarrow\rangle)$ in the $\mathbf{z}$ direction. In other word, the time of MR2 in non-polar state $(|\uparrow \downarrow\rangle)$ is very limited, which is in correspondence with the pressure-induced asymmetry of energies in polar and non-polar states in Fig. 2b. In Fig. 5e we show snapshots highlighted in red dots to verify conformational selection. The dipole moment of each rotor is shown in Supplementary Fig. 19. Difference of dipole moments perpendicular to surface is near to zero and oscillate in a small amplitude. This phenomenon indicates that their rotations are synchronous around the rotation axis. For dynamics parallel to the surface, the changes of dipole moments are simultaneous (see Supplementary Figs. 20-22). Therefore, bonding between rotors and stator is quite rigid and no other intrinsic degree of freedom can disturb the rotational reaction. When the electrodes retreat back to $7.3 \AA$, the flip stops and the dipole moment keeps negative during the next few picoseconds.

To confirm the frequency increase of MR2 under mechanical load, we analyze power spectral density (PSD) of dipole moment from DFTB-MD with fixed loading distances (see Supplementary Fig. 23). The distance of electrodes is fixed at $6.5 \AA$ and $6.7 \AA$, respectively, for a longer period of $15 \mathrm{ps}$. The PSD of the two cases is similar, except that a peak at $0.4 \mathrm{THz}$ is observed for distance of electrodes fixed at $6.5 \AA$. The frequency corresponds to the flip of polar rotors in MR2. For the case distance of electrode is fixed at $6.7 \AA$, no obvious peak corresponding to rotation is observed. It indicates that rotational frequency in $6.7 \AA$ case is not large enough for flip to happen in a 15 ps simulation. Thus, acceleration in rotational speed is achieved through increasing mechanical load.

Herein, the direction of dipole moment is not readily controlled by mechanical force. Due to the polarity of the rotors, electrical field offers an option to manipulate their directions ${ }^{17,40}$. Especially, combination of mechanical load and electric field improves the effect in manipulating polar rotors. When mechanical load is applied, the energy barrier is lowered significantly. Therefore, it is more apt for electric manipulation of polar rotors. For electric field perpendicular to anthracene, energy barrier increases for one state and decreases for the other, which creates discrepancy between $|\uparrow\rangle$ and $|\downarrow\rangle$. Hence, the orientation of polar rotor is controllable under different directions of electric field. If, on the contrary, no mechanical load is applied, the energy barrier is high and the electric field needed to flip the polar rotor increases largely. In this regard, mechanical force and electric field complement each other in manipulating polar rotors.

Minimizing the strength of electric field is helpful to molecular rotors for application in electronic devices. To understand the effects of electric field on these molecular rotors, we assess the frontier molecular orbitals of MR1-6 in different configurations. In $|\uparrow \uparrow\rangle$, frontier molecular orbitals are delocalized only within anthracene plane and transmission perpendicular to this plane is largely reduced by spatial regions on both sides (see Supplementary Fig. 24). Therefore, electrical field can operate on polar groups effectively in $|\uparrow \uparrow\rangle$. During the rotation, the delocalization of frontier molecular orbitals increases with reaction path from $|\uparrow\rangle$ to $|\rightarrow\rangle$. In $|\uparrow \rightarrow\rangle$, the frontier molecular orbitals extend through the axis to the polar rotor in TS (see Supplementary Fig. 25). In addition, delocalization increases on both polar rotors when both torsion angles decreases in the order of $90^{\circ}, 60^{\circ}$, and $30^{\circ}$ (see Supplementary Fig. 26). When the frontier molecular orbitals are delocalized, voltages on molecular rotors should be small enough a

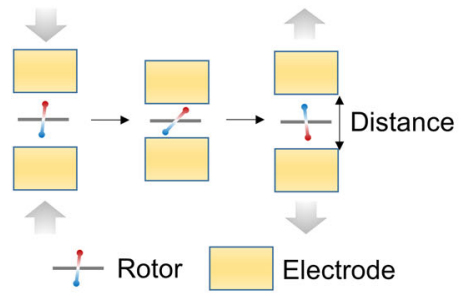

c

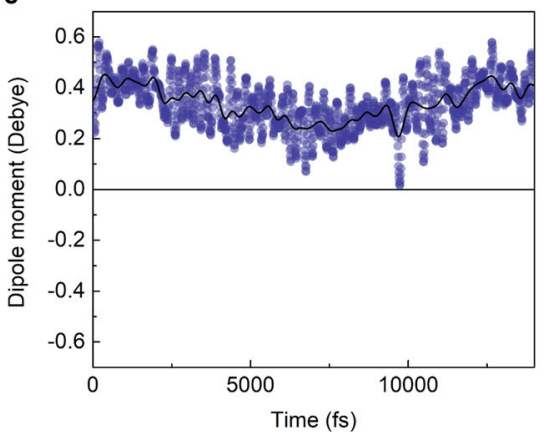

b

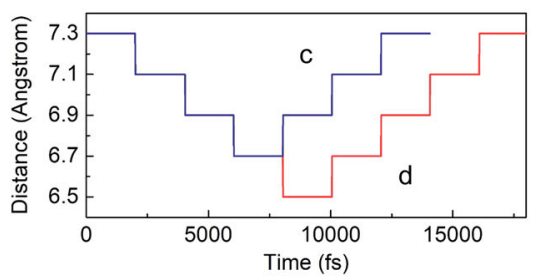

d

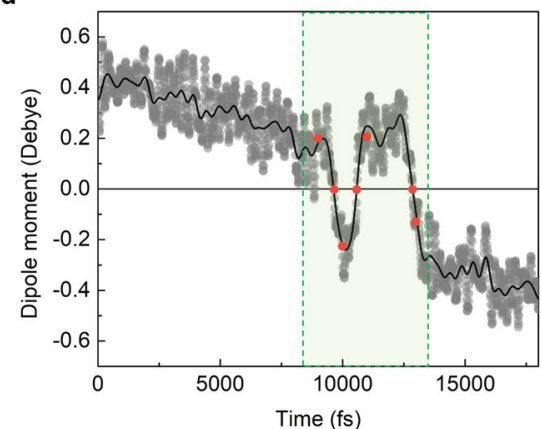

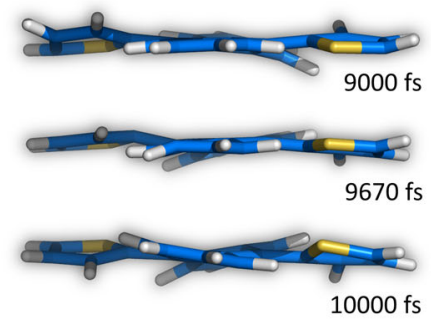
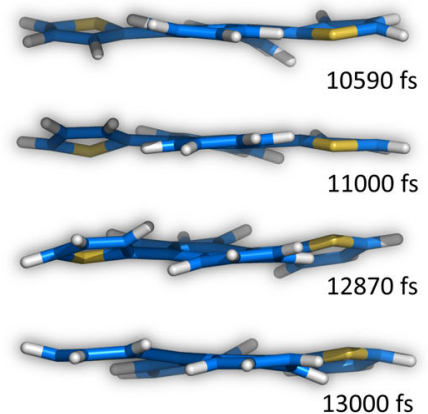

Fig. 5 Load-modulated rotor flip from density functional tight-binding molecular dynamics. a Illustration of mechanical loading process onto the molecular rotor. b The distance between the electrodes changes stepwise: for (c) it goes down from $7.3 \AA$ to $6.7 \AA$ and then back to $7.3 \AA$; for (d) it goes down further from $7.3 \AA$ to $6.5 \AA$ and then back to $7.3 \AA$. for trajectories. Dipole moment of rotor 2 diverges after 8 ps in (c) and (d). No dipole moment reverse is observed during the whole loading process in (c). In (d), the dipole moment reverses after several flips around zero in the region painted green. During the dynamic process, the snapshots of the rotor in red dots are shown in (e) with corresponding time. 
to exclude frontier molecular orbitals from bias window. Otherwise, resonant charge tunneling via frontier molecular orbitals may induce unexpected charging ${ }^{41}$ or even desorption ${ }^{42}$ of the molecular rotor. Charging alters the electronic structure of the molecular rotor, the dipole moment and consequently the forces induced by the electric field. Desorption of the molecular rotor destroys the contact between the molecule and the electrodes. Neither case is favored in constructing stable electronic devices. Accordingly, cooperation of mechanical force and electric field should be promising for modulating molecular rotor-based electronic devices.

\section{DISCUSSION}

We have designed molecular rotors with mechanically controllable rotational speed. Tremendous wide-range rotational speed is achieved under modulation of directional mechanical load. DFT and SCC-DFTB-MD calculations show the interplay between polar rotors and mechanical load pushes the rotors towards transition state and invokes acceleration. Meanwhile mechanical load induces energy asymmetry in different conformations, which is useful for creating polar state in multistate molecular rotors. Moreover, steric hindrance and polar effect play vital roles in the dependence rule of rotational energy barrier to the structure of polar rotor. This work presents a feasible avenue for achieving both rotational speed and thermal stability in molecular rotors, which can be applied to produce fast molecular switches.

\section{METHODS}

Free energy barrier of molecular rotors in gas phase

Density functional theory ${ }^{43}$ (DFT) calculations were employed to investigate the rotation energy barrier of designed molecular rotors. For gas phase molecular rotors, M06-2X functional ${ }^{44}$ with def2-TZVPD basis set ${ }^{45}$ was used in Gaussian software for equilibrium state and transition state calculations. Grimme's D3 dispersion corrections ${ }^{46}$ were used to improve London dispersion interactions. Reaction path was calculated with IRC approach $^{47,48}$ to confirm TS connected to $|\uparrow\rangle$ and $|\downarrow\rangle$. Electrostatic potential map and $\mathrm{NCl}$ was analyzed with Multiwfn ${ }^{49}$ and visualized in $\mathrm{VMD}^{50}$. Frequency of the molecule was achieved from the Hessian matrix and was corrected with a scaling factor of 0.97 for vibrational partition function. Partition function was calculated using hindered rotor density-ofstates (HRDS) interpolation approach ${ }^{51}$ to account internal rotation of molecular rotors. Free energy barrier $\Delta G^{\ddagger}$ and reaction rates $k$ was achieved from transition state theory using KiSThelP program ${ }^{52}$ :

$\Delta G^{\ddagger}=\Delta E^{\ddagger} \ln \frac{Q^{\top S}(T)}{Q^{\mathrm{R}}(T)}$

$k=\chi(T) \sigma \frac{k_{\mathrm{B}} T}{h} \mathrm{e}^{-\Delta G^{\ddagger} / k_{\mathrm{B}} T}=\chi(T) \sigma \frac{k_{\mathrm{B}} T}{h} \frac{Q^{\top S}(T)}{Q^{\mathrm{R}}(T)} \mathrm{e}^{-\Delta E^{\ddagger} / k_{\mathrm{B}} T}$,

where $\Delta E^{\ddagger}$ is the difference in DFT calculation between transition state and reactant, $Q^{T S}$ and $Q^{R}$ denote the total partition functions of the transition state and reactant, $X(T)$ is the transmission coefficient from Wigner tunneling correction ${ }^{53}, \sigma$ is the reaction degeneracy, $k_{\mathrm{B}}$ is Boltzmann's constant, $T$ is the temperature and $h$ is Planck's constant.

Free energy barrier of molecular rotor under mechanical load For molecular rotors under mechanical load, PBE functional ${ }^{54}$ with $400 \mathrm{eV}$ energy cutoff was employed in VASP ${ }^{55}$. DFT-D3 method with BeckeJohnson damping correction ${ }^{56}$ was used to account for London dispersion interactions. The calculation was done in tetragonal superlattices using periodic boundary condition (see Supplementary Fig. 9). The anthracene stator was parallel to $20 \times 20 \AA^{2}$ square base. For the lattice perpendicular to the anthracene stator, the lattice constant $c$ was varied among $6,4,3.5$, and $3 \AA$ to simulate different loads in this direction. At specific intermolecular distance, mechanical load on molecular rotor in $|\uparrow \uparrow\rangle$ and corresponding free energy barrier were calculated. Transition state and reaction path were determined using climbing image nudged elastic band
(CI-NEB) method ${ }^{57,58}$. For each molecular rotor, we constructed nine images along reaction path. Carbon atoms 4a, 8a, 9, 9a, 10, and 10a (see Fig. 4a) were fixed to prevent rotation of anthracene. All the other atoms were relaxed until force was smaller than $0.01 \mathrm{eV} \cdot \AA^{-1}$. Free energy barrier $\Delta G^{\ddagger}$ and reaction rates $k$ were obtained similarly as in gas phase. For vibrational partition function of all relaxed structures, frequency was calculated with finite difference of $0.015 \AA$.

For molecular rotors adsorbed on Au(111), PBE functional with $400 \mathrm{eV}$ energy cutoff was employed in VASP. Three layers of $5 \times 5 \mathrm{Au}(111)$ surface with the bottom layer fixed were used as the surface. After the adsorption of molecular rotor, a vacuum layer of $20 \AA$ was added to reduce errors from PBC. All other atoms were relaxed until force was smaller than $0.01 \mathrm{eV} \AA^{-1}$.

Evolution of molecular rotor under mechanical load

Self-consistent charge density functional tight-binding molecular dynamics ${ }^{59,60}$ (SCC-DFTB-MD) was employed to simulate the evolution of MR2 under mechanical load using DFTB + code $^{61}$. DFT-D3 method with Becke-Johnson damping correction was used to account for London dispersion. Simulations were carried out at $500 \mathrm{~K}$ and Nosé-Hoover thermostat chains were attached to the system. A time-step of $1 \mathrm{fs}$ was used and data was achieved every $10 \mathrm{fs}$. Three layers of $5 \times 5 \mathrm{Au}(111)$ surface were used as top and bottom electrodes between which the molecular rotor was adsorbed. At the beginning, the distance of the two electrodes was fixed at a distance of $7.5 \AA$ for structural relaxation. Then the top electrode was moved $0.2 \AA$ towards the bottom electrode every 2 ps until the distance reached $6.5 \AA$ (case I) or $6.7 \AA$ (case II). Then the top electrode was moved back to $7.5 \AA$ at the same speed. Five trajectories were obtained for each loading scheme. For case I, flip of polar rotor was observed for all trajectories. Among them, two trajectories ended up with $|\uparrow \uparrow\rangle$ state, two ended up with $|\downarrow \downarrow\rangle$ and one ended up with $|\uparrow \downarrow\rangle$. For case II, no flip of polar rotor was observed for all trajectories. For PSD analysis, the distance between electrodes was fixed at $6.5 \AA$ and $6.7 \AA$ respectively in simulation time of $15 \mathrm{ps}$.

\section{DATA AVAILABILITY}

All data needed to evaluate the conclusions in the paper are present in the paper and/or the supplementary information. Additional data related to this paper are available from the corresponding author upon reasonable request.

\section{CODE AVAILABILITY}

Multiwfn (sobereva.com/multiwfn), VMD (www.ks.uiuc.edu/Research/vmd), KiSThelP (kisthelp.univ-reims.fr) and DFTB + (dftbplus.org) are open-source codes, freely available from their websites. Post-processing codes are available from the corresponding author upon reasonable request.

Received: 25 May 2020; Accepted: 6 November 2020; Published online: 07 December 2020

\section{REFERENCES}

1. Erbas-Cakmak, S., Leigh, D. A., McTernan, C. T. \& Nussbaumer, A. L. Artificial molecular machines. Chem. Rev. 115, 10081-10206 (2015).

2. Bauer, J., Hou, L., Kistemaker, J. C. \& Feringa, B. L. Tuning the rotation rate of lightdriven molecular motors. J. Org. Chem. 79, 4446-4455 (2014).

3. Faulkner, A., van Leeuwen, T., Feringa, B. L. \& Wezenberg, S. J. Allosteric regulation of the rotational speed in a light-driven molecular motor. J. Am. Chem. Soc. 138, 13597-13603 (2016).

4. Greb, L. \& Lehn, J. M. Light-driven molecular motors: imines as four-step or twostep unidirectional rotors. J. Am. Chem. Soc. 136, 13114-13117 (2014).

5. Klok, M., Browne, W. R. \& Feringa, B. L. Kinetic analysis of the rotation rate of lightdriven unidirectional molecular motors. Phys. Chem. Chem. Phys. 11, 9124-9131 (2009).

6. Bandara, H. M. D. \& Burdette, S. C. Photoisomerization in different classes of azobenzene. Chem. Soc. Rev. 41, 1809-1825 (2012).

7. Tsurunaga, M. et al. Dielectric relaxation of powdered molecular gyrotops having a thiophene dioxide-diyl as a dipolar rotor. Org. Lett. 20, 6934-6937 (2018).

8. Sun, Y. Z. et al. Plastic crystals with polar halochromate anion: thermosensitive dielectrics based upon plastic transition and dipole rotation. Inorg. Chem. 55, 11418-11425 (2016). 
9. Kobr, L. et al. Inclusion compound based approach to forming arrays of artificial dipolar molecular rotors: a search for optimal rotor structures. Adv. Mater. 25, 443-448 (2013).

10. Horinek, D. \& Michl, J. Surface-mounted altitudinal molecular rotors in alternating electric field: single-molecule parametric oscillator molecular dynamics. Proc. Natl Acad. Sci. U. S. A. 102, 14175-14180 (2005)

11. Zheng, X. et al. Dipolar and nonpolar altitudinal molecular rotors mounted on an Au(111) surface. J. Am. Chem. Soc. 126, 4540-4542 (2004).

12. Magnera, T. F. \& Michl, J. Altitudinal surface-mounted molecular rotors. Top. Curr. Chem. 262, 63-97 (2005).

13. Stacko, P. et al. Locked synchronous rotor motion in a molecular motor. Science 356, 964-968 (2017).

14. Iwamura, H. \& Mislow, K. Stereochemical consequences of dynamic gearing. Acc. Chem. Res. 21, 175-182 (1988).

15. Simpson, C. D. et al. Nanosized molecular propellers by cyclodehydrogenation of polyphenylene dendrimers. J. Am. Chem. Soc. 126, 3139-3147 (2004).

16. Wang, B. \& Král, P. Chemically tunable nanoscale propellers of liquids. Phys. Rev. Lett. 98, 266102 (2007).

17. Meng, L. et al. Side-group chemical gating via reversible optical and electric control in a single molecule transistor. Nat. Commun. 10, 1450 (2019).

18. Crespi, S., Simeth, N. A. \& König, B. Heteroaryl azo dyes as molecular photoswitches. Nat. Rev. Chem. 3, 133-146 (2019).

19. $\mathrm{Wu}, \mathrm{D}$. et al. Fluorescent chemosensors: the past, present and future. Chem. Soc. Rev. 46, 7105-7123 (2017)

20. Donhauser, Z. J. et al. Conductance switching in single molecules through conformational changes. Science 292, 2303-2307 (2001).

21. Tierney, H. L. et al. Experimental demonstration of a single-molecule electric motor. Nat. Nanotechnol. 6, 625-629 (2011).

22. Guo, F. S. et al. Magnetic hysteresis up to 80 kelvin in a dysprosium metallocene single-molecule magnet. Science 362, 1400-1403 (2018).

23. Vicario, J., Meetsma, A. \& Feringa, B. L. Controlling the speed of rotation in molecular motors. Dramatic acceleration of the rotary motion by structural modification. Chem. Commun. 116, 5910-5912 (2005).

24. Kudernac, T. et al. Electrically driven directional motion of a four-wheeled molecule on a metal surface. Nature 479, 208-211 (2011).

25. Goswami, A., Saha, S., Biswas, P. K. \& Schmittel, M. Nanomechanical motion triggered by metal coordination: from functional devices to networked multicomponent catalytic machinery. Chem. Rev. 120, 125-199 (2019).

26. Inukai, M. et al. Control of molecular rotor rotational frequencies in porous coordination polymers using a solid-solution approach. J. Am. Chem. Soc. 137, 12183-12186 (2015).

27. $\mathrm{Wu}, \mathrm{Y}$. et al. A multistage rotational speed changing molecular rotor regulated by $\mathrm{pH}$ and metal cations. Nat. Commun. 9, 1953 (2018).

28. Tang, Y. Y. et al. A multiaxial molecular ferroelectric with highest Curie temperature and fastest polarization switching. J. Am. Chem. Soc. 139, 13903-13908 (2017).

29. Tang, Y. Y. et al. Ultrafast polarization switching in a biaxial molecular ferroelectric thin film: [Hdabco]ClO4. J. Am. Chem. Soc. 138, 15784-15789 (2016).

30. Sun, Z. et al. A molecular ferroelectric showing room-temperature record-fast switching of spontaneous polarization. Angew. Chem. Int. Ed. Engl. 57, 9833-9837 (2018).

31. Taft, R. W. The separation of relative free energies of activation to three basic contributing factors and the relationship of rhese to structure. J. Am. Chem. Soc. 75, 4534-4537 (1953).

32. Johnson, E. R. et al. Revealing noncovalent interactions. J. Am. Chem. Soc. 132, 6498-6506 (2010).

33. Matsuura, T., Nakajima, M. \& Shimoyama, Y. Growth of self-assembled monolayer of thiophene on gold surface: an infrared spectroscopic study. Jpn. J. Appl. Phys. 40, 6945-6950 (2001).

34. Sako, E. O. et al. Reactive adsorption of thiophene on $\mathrm{Au}(111)$ from solution. Chem. Phys. Lett. 413, 267-271 (2005).

35. Ito, E., Noh, J. \& Hara, M. Adsorption states and thermal desorption behaviors of thiophene derivative self-assembled monolayers on $\mathrm{Au}(111)$. Surf. Sci. 602, 3291-3296 (2008).

36. Zhou, J. et al. Electronic structure of the thiophene/Au(111) interface probed by two-photon photoemission. J. Phys. Chem. B 114, 13670-13677 (2010).

37. Durso, M. et al. Synthesis by MW-assisted direct arylation, side-arms driven selfassembly and functional properties of 9,10-dithienylanthracene orthogonal materials. Tetrahedron 70, 6222-6228 (2014).

38. Gray, V. et al. Photophysical characterization of the 9,10-disubstituted anthracene chromophore and its applications in triplet-triplet annihilation photon upconversion. J. Mater. Chem. C. 3, 11111-11121 (2015).
39. Muñoz-Pérez, J. et al. Raman and surface enhanced Raman scattering study of the orientation of cruciform 9,10-anthracene thiophene and furan derivatives deposited on a gold colloidal surface. J. Mol. Struct. 1122, 198-204 (2016).

40. Zhang, Y. et al. A chiral molecular propeller designed for unidirectional rotations on a surface. Nat. Commun. 10, 3742 (2019).

41. Galperin, M., Ratner, M. A., Nitzan, A. \& Troisi, A. Nuclear coupling and polarization in molecular transport junctions: beyond tunneling to function. Science 319, 1056-1060 (2008).

42. Seideman, T. Current-driven dynamics in molecular-scale devices. J. Phys.: Condens. Matter 15, R521-R549 (2003).

43. Kohn, W. \& Sham, L. J. Self-consistent equations including exchange and correlation effects. Phys. Rev. 140, A1133-A1138 (1965).

44. Zhao, Y., Schultz, N. E. \& Truhlar, D. G. Design of density functionals by combining the method of constraint satisfaction with parametrization for thermochemistry, thermochemical kinetics, and voncovalent interactions. J. Chem. Theory Comput. 2, 364-382 (2006).

45. Pritchard, B. P. et al. New basis set exchange: an open, up-to-date resource for the molecular sciences community. J. Chem. Inf. Model. 59, 4814-4820 (2019).

46. Grimme, S., Antony, J., Ehrlich, S. \& Krieg, H. A consistent and accurate ab initio parametrization of density functional dispersion correction (DFT-D) for the 94 elements H-Pu. J. Chem. Phys. 132, 154104 (2010).

47. Fukui, K. Formulation of the reaction coordinate. J. Phys. Chem. 74, 4161-4163 (1970).

48. Fukui, K. The path of chemical reactions - the IRC approach. Acc. Chem. Res. 14, 363-368 (1981).

49. Lu, T. \& Chen, F. Multiwfn: a multifunctional wavefunction analyzer. J. Comput. Chem. 33, 580-592 (2012).

50. Humphrey, W., Dalke, A. \& Schulten, K. VMD: visual molecular dynamics. J. Mol. Graph. 14, 33-38 (1996).

51. McClurg, R. B., Flagan, R. C. \& Goddard, W. A. III The hindered rotor density-ofstates interpolation function. J. Chem. Phys. 106, 6675-6680 (1997).

52. Canneaux, S., Bohr, F. \& Henon, E. KiSThelP: a program to predict thermodynamic properties and rate constants from quantum chemistry results. J. Comput. Chem. 35, 82-93 (2014).

53. Wigner, E. Über das überschreiten von potentialschwellen bei chemischen reaktionen [Crossing of potential thresholds in chemical reactions]. Z. Phys. Chem. 19B, 203-216 (1932).

54. Perdew, J. P., Burke, K. \& Ernzerhof, M. Generalized gradient approximation made simple. Phys. Rev. Lett. 77, 3865-3868 (1996).

55. Kresse, G. \& Hafner, J. Ab initio molecular dynamics for liquid metals. Phys. Rev. $B$ 47, 558-561 (1993).

56. Grimme, S., Ehrlich, S. \& Goerigk, L. Effect of the damping function in dispersion corrected density functional theory. J. Comput. Chem. 32, 1456-1465 (2011).

57. Henkelman, G., Uberuaga, B. P. \& Jónsson, H. A climbing image nudged elastic band method for finding saddle points and minimum energy paths. J. Chem. Phys. 113, 9901-9904 (2000).

58. Henkelman, G. \& Jónsson, H. Improved tangent estimate in the nudged elastic band method for finding minimum energy paths and saddle points. J. Chem. Phys. 113, 9978-9985 (2000).

59. Elstner, M. et al. Self-consistent-charge density-functional tight-binding method for simulations of complex materials properties. Phys. Rev. B 58, 7260-7268 (1998).

60. Fihey, A. et al. SCC-DFTB parameters for simulating hybrid gold-thiolates compounds. J. Comput. Chem. 36, 2075-2087 (2015).

61. Hourahine, B. et al. DFTB +, a software package for efficient approximate density functional theory based atomistic simulations. J. Chem. Phys. 152, 124101 (2020).

\section{ACKNOWLEDGEMENTS}

This study was supported by the National Natural Science Foundation of China (NSFC) (Grants 11972383, 11672339 and 81827802), and the Guangzhou Science and Technology key Project (Grant 201707020002). Y.Z. also acknowledged support from the National Youth Talent Support Program and Guangdong Natural Science Funds for Distinguished Young Scholar.

\section{AUTHOR CONTRIBUTIONS}

Y.Z. initiated and performed this work and manuscript. X.Y.Z. and J.S. conceived and designed the basic idea and structures. J.S. performed the simulations. X.Y.Z. and J.S. co-wrote the manuscript. All authors contributed to the discussion and reviewed the manuscript. 


\section{COMPETING INTERESTS}

The authors declare no competing interests.

\section{ADDITIONAL INFORMATION}

Supplementary information is available for this paper at https://doi.org/10.1038/ s41524-020-00457-6.

Correspondence and requests for materials should be addressed to X.Z. or Y.Z.

Reprints and permission information is available at http://www.nature.com/reprints

Publisher's note Springer Nature remains neutral with regard to jurisdictional claims in published maps and institutional affiliations.
Open Access This article is licensed under a Creative Commons Attribution 4.0 International License, which permits use, sharing, adaptation, distribution and reproduction in any medium or format, as long as you give appropriate credit to the original author(s) and the source, provide a link to the Creative Commons license, and indicate if changes were made. The images or other third party material in this article are included in the article's Creative Commons license, unless indicated otherwise in a credit line to the material. If material is not included in the article's Creative Commons license and your intended use is not permitted by statutory regulation or exceeds the permitted use, you will need to obtain permission directly from the copyright holder. To view a copy of this license, visit http://creativecommons. org/licenses/by/4.0/.

(c) The Author(s) 2020 